\title{
NLTE analysis of Sr lines in spectra of late-type stars with new R-matrix atomic data ${ }^{\star} \star \star$
}

\author{
M. Bergemann ${ }^{1}$, C. J. Hansen ${ }^{2}$, M. Bautista ${ }^{3}$, and G. Ruchti ${ }^{1}$ \\ ${ }^{1}$ Max-Planck Institute for Astrophysics, Karl-Schwarzschild Str. 1, 85741 Garching, Germany \\ e-mail: mbergema@mpa-garching.mpg.de \\ ${ }^{2}$ Landessternwarte, Königstuhl 12, 69117 Heidelberg, Germany \\ 3 Department of Physics, Western Michigan University, Kalamazoo, MI 49008, USA
}

Received 13 April 2012 / Accepted 5 July 2012

\section{ABSTRACT}

\begin{abstract}
We investigate the statistical equilibrium of neutral and singly-ionized strontium in late-type stellar atmospheres. Particular attention is given to the completeness of the model atom, which includes new energy levels, transition probabilities, photoionization and electron-impact excitation cross-sections computed with the R-matrix method. The NLTE model is applied to the analysis of Sr I and Sr II lines in the spectra of the Sun, Procyon, Arcturus, and HD 122563, showing a significant improvement in the ionization balance compared to LTE line formation calculations, which predict abundance discrepancies of up to 0.5 dex. The solar $\mathrm{Sr}$ abundance is $\log \epsilon=2.93 \pm 0.04 \mathrm{dex}$, in agreement with the meteorites. We present the grid of NLTE abundance corrections for Sr I and Sr II lines that covers a wide range of stellar parameters.
\end{abstract}

Key words. atomic data - line: formation - radiative transfer - Sun: abundances - stars: abundances - stars: atmospheres

\section{Introduction}

Spectroscopic observations of low-mass stars have shaped our understanding of the Galactic evolution and stellar nucleosynthesis. Strontium, as one of the abundant r-process elements, has been extensively investigated in the past few decades. However, its main production site has not yet been identified: the observed abundances of $\mathrm{Sr}$ in metal-poor stars are far too large to be explained by conventional rapid neutron-capture nucleosynthesis in SNe II, suggesting some alternative exotic scenarios, such as the light element primary process (Travaglio et al. 2004), rp-process in accretion disks around low-mass black holes (Schatz et al. 1998), black hole-neutron star mergers (Surman et al. 2008), high-entropy winds in SN II (Farouqi et al. 2010), and low-mass electron-capture supernovae (Wanajo et al. 2011), to name just a few (see José \& Iliadis 2011, and references therein).

Until recently, determinations of $\mathrm{Sr}$ abundances in metalpoor stars relied almost exclusively on the two near-UV lines of SrII, which are sufficiently strong to be detected also in the spectra of moderate-to-low resolution and signal-to-noise ratio. The drawback is that in spectra of stars that are typically used for studies of Galactic chemical evolution, $[\mathrm{Fe} / \mathrm{H}]>-2$, these lines saturate and develop pronounced damping wings, overlapping with various atomic and molecular blends. Therefore, the weak Sr I line at $4607.34 \AA$ and/or the Sr II line at $4161 \AA$ are sometimes preferred at higher metallicities. However, there is evidence that the SrI lines may be subject to non-local thermodynamic equilibrium (hereafter, NLTE) effects (Barklem \& O'Mara 2000), which has been supported by ab initio

\footnotetext{
* Tables 3-5 are available in electronic form at http://www . aanda.org

$\star \star$ The atomic data is available at the CDS via anonymous ftp to cdsarc.u-strasbg.fr $(130.79 .128 .5)$ or via http://cdsarc.u-strasbg.fr/viz-bin/qcat?J/A+A/546/A90
}

calculations solving for radiative transfer in NLTE for a small grid or red giant model atmospheres (Short \& Hauschildt 2006). In a few studies that used near-IR spectra, the Sr II triplet (10 037, 10327, and $10915 \AA$ have also been used (Andrievsky et al. 2011). In the majority of published studies, the preference was given to one ionization stage only (e.g., Jehin et al. 1999), and only a few studies investigated both Sr I and Sr II lines (Gratton \& Sneden 1994; Cowan et al. 2002), finding discrepant results.

In this study, we perform for the first time an NLTE analysis of the SrI and Sr II lines in spectra of late-type stars. The new atomic model was constructed from state-of-art atomic data that were computed specifically for this work. The NLTE model atom was tested on several reference stars with parameters determined by other independent methods. Furthermore, we present a large grid of NLTE abundance corrections for Sr I and Sr II lines. The results presented in this work will be applied to the analysis of a representative sample of metal-poor stars observed at a very high resolution and signal-to-noise ratio (in Hansen et al., in prep.) Furthermore, we plan to undertake an NLTE Sr abundance analysis of the thick-disk and halo stars with spectroscopic parameters from Ruchti et al. (2011).

\section{Methods}

\subsection{Model atmospheres and NLTE calculations}

All calculations in this work were performed with classical 1D LTE plane-parallel model atmospheres. We used the MAFAGSOS (Grupp 2004a,b) and MAFAGS-ODF models, which are well-adapted for the analysis of late-type stars. A brief description of these models and comparison with other models of a similar type (MARCS, Gustafsson et al. 2008) is presented in Bergemann et al. (2012).

The NLTE statistical equilibrium calculations were performed with the revised version of the DETAIL code 
(Butler \& Giddings 1985). The statistical equilibrium and radiative transfer equations were solved by the accelerated $\Lambda$-iteration method in the formulation of Rybicki \& Hummer (1991, 1992). The method allows for self-consistent treatment of overlapping lines and continua. A description of the code with some recent modifications related to the treatment of background line opacity can be found in Bergemann et al. (2012). In the statistical equilibrium calculations, we treated the diagnostic lines investigated here with Voigt profiles, while all other Sr lines were computed with a Gaussian profile with 13 frequency points.

The LTE and NLTE abundances for the reference stars, as well as the NLTE abundance corrections, were determined with a full spectrum synthesis with the revised version of the SIU code (Reetz 1999), which was adapted for automated NLTE abundance calculations (Sbordone, priv. comm.). The line lists in SIU have been continuously updated by the members of the Munich group (Gehren et al. 2004, 2006) and they are mainly based on the Kurucz ${ }^{1}$, Hannover ${ }^{2}$, and NIST (Ralchenko et al. 2012) compilations.

\subsection{Model atom}

The model atom of Sr was constructed using the available atomic data from the Hannover and NIST databases that were supplemented by our new calculations ${ }^{3}$ of atomic energy levels, dipolepermitted and forbidden transitions (see below). Thus, the model is the most complete representation of atomic system of Sr I and Sr II, to date. In particular, we also included $135 \mathrm{Sr}$ II transitions from the Coulomb approximation calculations of Lindgård $\&$ Nielson (1977). The neutral atom is, thus, represented by 141 levels with the principle quantum numbers $n * \leq 20$, and the uppermost level $20 \mathrm{f}^{1} \mathrm{~F}^{\circ}$ is located at $5.66 \mathrm{eV}$, i.e. $0.03 \mathrm{eV}$ from the first ionization limit at $5.69 \mathrm{eV}$. The singly-ionized atom includes 49 levels including the highest experimentally observed level $14 \mathrm{~d}^{2} \mathrm{D}$, which is located at $10.684 \mathrm{eV}$. The model is closed by the Sr III ground state $4 \mathrm{p}^{6}{ }^{1} \mathrm{~S}_{0}$. The number of dipole-allowed transitions is 336 and 214 for Sr I and Sr II, respectively.

Our model of $\mathrm{Sr}$ is similar to that of Andrievsky et al. (2011) with respect to the term structure and the number of dipolepermitted transitions of Sr II. However, we included more accurate transition probabilities for Sr II. More importantly, our model atom includes new quantum-mechanical photoionization cross-sections for Sr I and $\mathrm{e}^{-}$impact excitation cross-sections for dipole-permitted and forbidden transitions in Sr II (see below). The model atoms employed by Short \& Hauschildt (2006) and Mashonkina \& Gehren (2001) are much simpler than those employed in this work (84 and 41 total levels, respectively).

\subsubsection{New calculations of atomic data}

We computed single-electron orbitals for the target ions ${ }^{4} \mathrm{Sr}^{+}$ with the atomic structure code autostructure by Badnell (1986, 1997). This code is an extension of the program superstructure

\footnotetext{
1 kurucz.harvard.edu/

2 www.pmp.uni-hannover.de/cgi-bin/ssi/test/kurucz/ sekur.html. The atomic line data used in this database are taken from Kurucz \& Bell (1995).

3 The new atomic data for $\mathrm{Sr}$ presented here and in Bautista et al. (2002), including the energy levels, $f$-values, photoionization, and eimpact excitation cross-sections, can be provided upon request.

4 We note that different notations are standard for atomic physics and spectroscopy, and we chose to respect this difference in our work. Ionization stage of an element is thus indicated by a numerical superscript in Sect. 2.2.1 describing the atomic physics calculations and by a roman numerical in astrophysical application in the other sections.
}
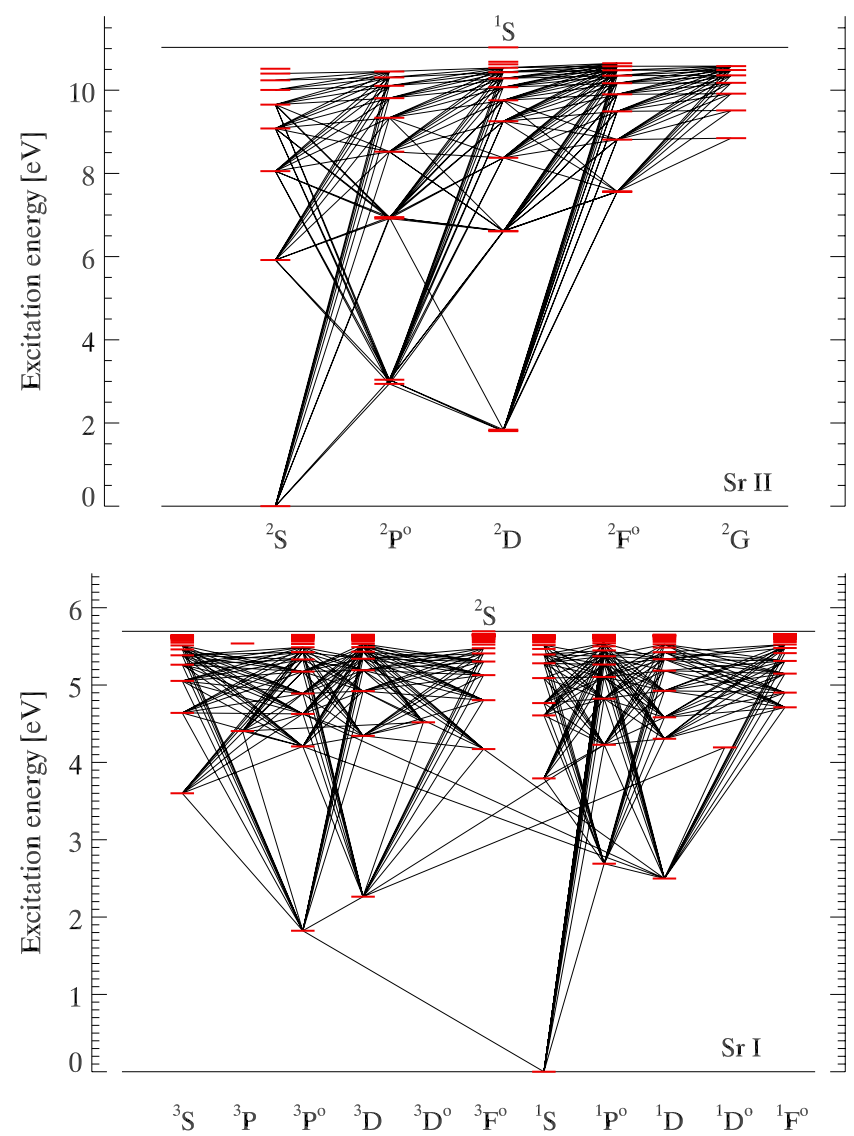

Fig. 1. Grotrian diagram of the Sr I (bottom) and Sr II (top) model atoms. Only dipole-allowed transitions are shown. This is the most complete model atom used for NLTE calculations of FGK stars to date.

(Eissner \& Nussbaumer 1969) and computes fine-structure CI energy levels and radiative and Auger rates in a Breit-Pauli relativistic framework. Single electron orbitals are constructed by diagonalizing the non-relativistic Hamiltonian, within a statistical Thomas-Fermi-Dirac-Amaldi (TFDA) model potential (Eissner \& Nussbaumer 1969). The scaling parameters are optimized by minimizing a weighted sum of the LS term energies. We employed a very extensive configuration expansion with configurations of the form $4 \mathrm{p}^{6} \mathrm{nl}$, with $n$ going from 4 to 6 and $l \leq 3$, and configurations with multiple promotion from the $4 \mathrm{p}$ orbital, i.e. $4 \mathrm{p}^{q} 5 \mathrm{~s}^{r} 4 \mathrm{~d}^{t} n l$ with $3 \leq q \leq 6,0 \leq r \leq 2$, and $0 \leq t \leq 2$. These multiple promotions from the $4 \mathrm{p}$ orbital are essential for computing the photoionization cross sections, which will be the subject of a future publication.

Our target representations give term energies in reasonable agreement, within $\sim 5$ percent with experimental values.

The photoionization cross-sections were computed with the R-matrix method (Burke et al. 1971). The present calculation for $\mathrm{Sr}^{0}$ includes the lowest $67 \mathrm{LS}$-terms of the target in the close coupling expansion and all short range $(N+1)$-electron configurations that result from adding an electron to the target configurations. We found 197 singlet and triplet bound terms of $\mathrm{Sr}^{0}$ with $n<9$ and $L \leq 5$, and computed photoionization cross sections for all of them. The cross sections were computed at an even energy mesh of $5 \times 10^{-5}$ Ry from threshold up to $0.23 \mathrm{Ry}$, mesh of $10^{-3}$ Ry from 0.23 Ry to $2.3 \mathrm{Ry}$ (roughly the highest target threshold), and another mesh of 0.02 Ry from $2.3 \mathrm{Ry}$ to $4.3 \mathrm{Ry}$. Figure 2 shows the partial cross sections for ionization from the ground term of $\mathrm{Sr}^{0}$ into each of the first six terms of the target $\mathrm{Sr}^{+}$. We note, however, that DETAIL is not 
M. Bergemann et al.: Statistical equilibrium of Sr in late-type stars

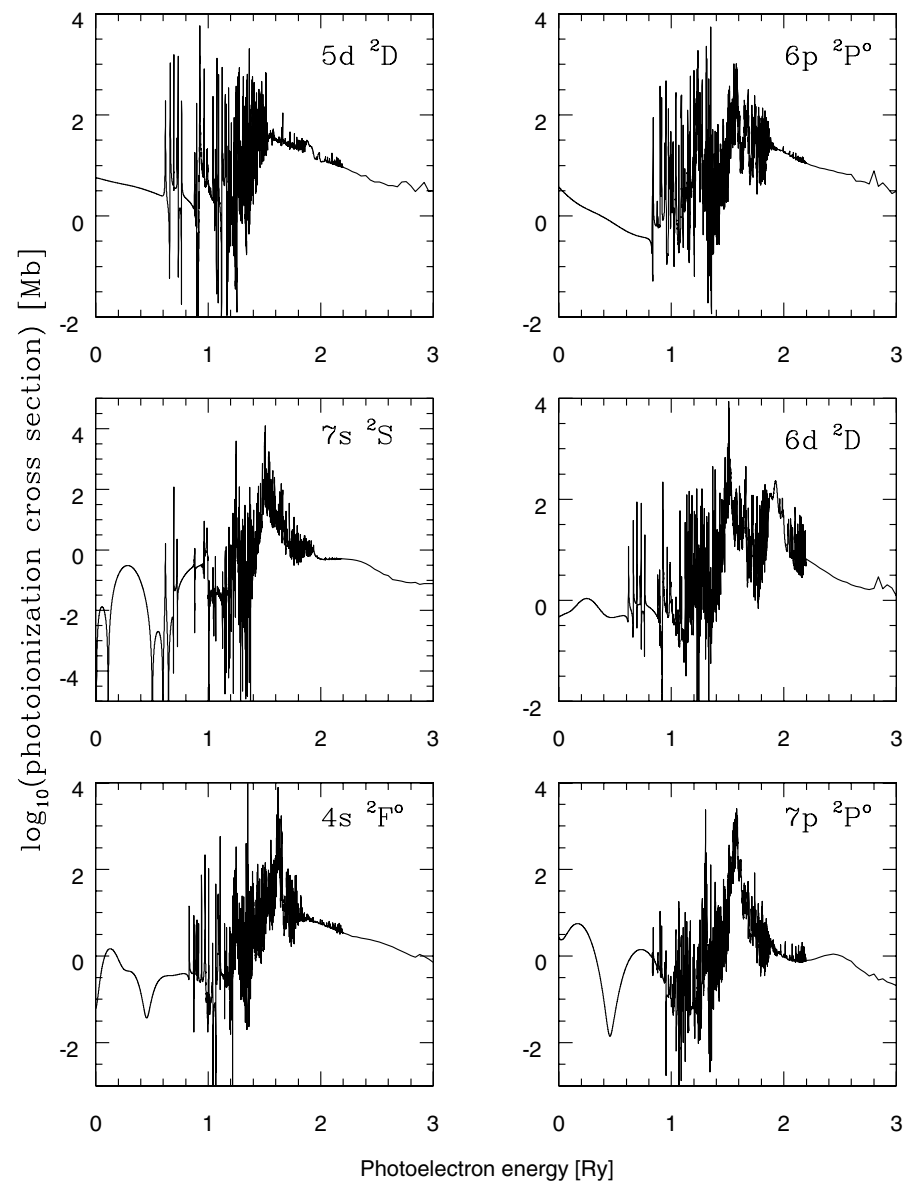

Fig. 2. Partial photoionization cross-sections for the Sr I ground state into the lowest six states of the Sr II target. The cross-sections are dominated by prominent resonances.

yet able to consistently treat ionization to specific states of the target ion. Therefore, total photoionization cross-sections were adopted here. Figure 3 shows the total cross sections for the lowest six excited state of $\mathrm{Sr}^{0}$. For comparison, we show the hydrogenic cross-sections computed with the effective principal quantum number. The latter reproduces the functional dependence of the cross-section with energy. However, the quantummechanical photoionization cross-sections are typically larger both in the background (factor of two to five) and in the resonances (up to three orders of magnitude).

\subsubsection{Collision rates}

Electron impact excitation rate coefficients for the 49 levels of Sr II levels were taken from Bautista et al. (2002). The data were computed with the same technique, i.e., the close-coupling approximation with the R-matrix method. For all other levels of Sr I and Sr II the electron collision rates were computed using the formulae of van Regemorter (1962) for dipole-allowed and Allen (1973) for dipole-forbidden transitions. Cross-sections for transitions caused by inelastic collisions with H I atoms are basically unknown for any atom heavier than $\mathrm{Mg}$. The only available formula developed originally for collisions between equal $\mathrm{H}$-like atoms (Drawin 1968, 1969) that was subsequently slightly modified by Steenbock \& Holweger (1984) and Lambert (1993) was shown to overestimate the rates of bound-bound transitions by two to seven orders of magnitude (Belyaev \& Barklem 2003; Barklem et al. 2012). Furthermore, the charge transfer processes

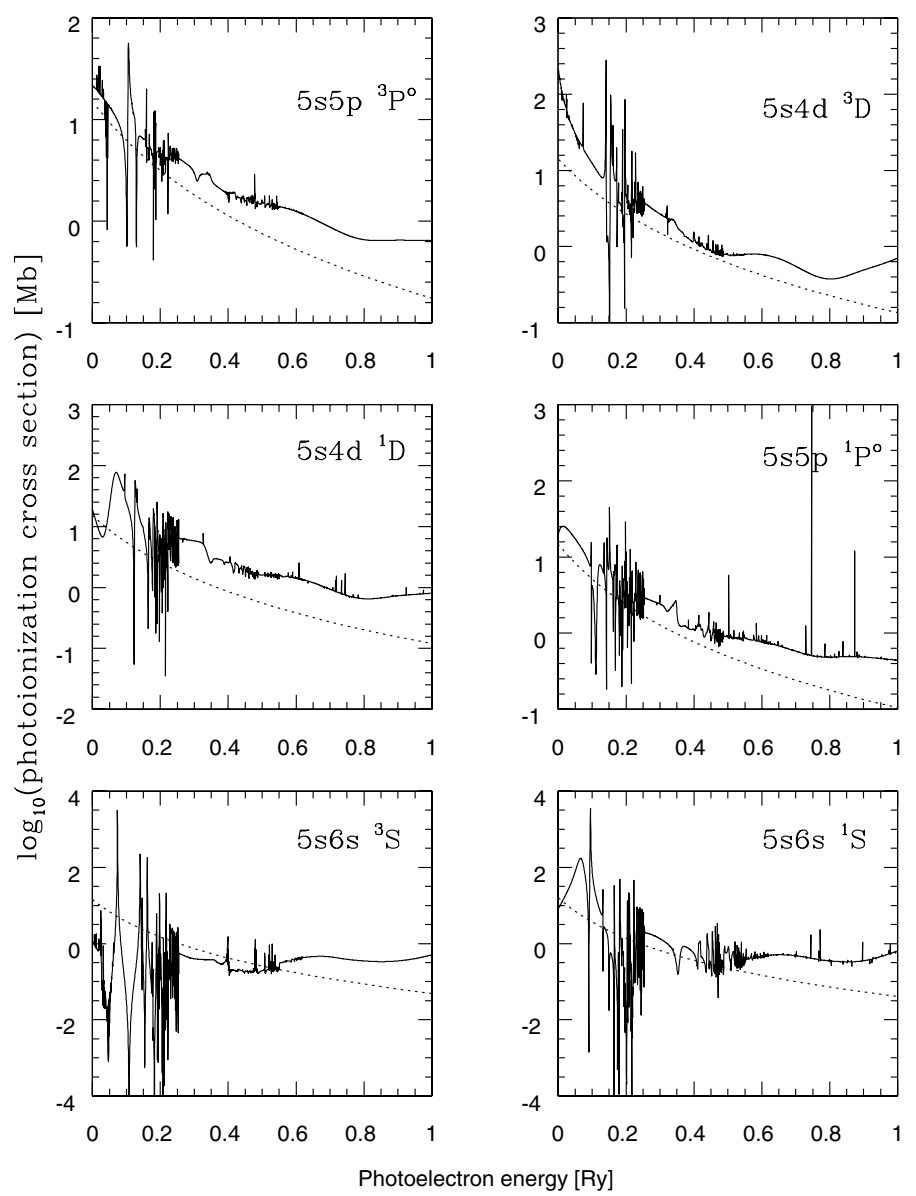

Fig. 3. Total photoionization cross-sections for the selected Sr I levels. Dotted lines show hydrogenic cross-sections. The new cross-sections are up to few orders of magnitude larger than the commonly used hydrogenic approximation.

cannot be described by this simple classical formula at all. Our tests with various scaling factors to the Drawin H I inelastic collision cross-sections demonstrated that the excitation and ionization balance of $\mathrm{SrI} / \mathrm{Sr}$ II in the reference stars can be satisfied simultaneously only if the efficiency of $\mathrm{H}$ I collisions is very low (Sect. 4), several orders of magnitude lower than that given by the Drawin recipe, which is consistent with the quantummechanical results mentioned above. On these grounds we did not include inelastic $\mathrm{H}$ I collisions in our reference model atom.

\subsection{Line selection and atomic data}

In spectra of FGK stars, only a few Sr lines are useful for abundance determinations. These are the two SrI and 6 Sr II lines. The SrI resonance line at $4607 \AA$ is weak, and therefore sufficiently reliable for more metal-rich stars $([\mathrm{Fe} / \mathrm{H}]>-1.5)$ observed at high resolution. The two resonance $\mathrm{Sr}$ II lines, in contrast, remain strong even in spectra of very metal-poor stars, but severe blends in the inner and outer wings lead to systematic errors in abundances if the blends are not properly accounted for. The three near-IR Sr II lines at $1 \mu \mathrm{m}$ appear to be unblended and are sufficiently strong to be detected even at $[\mathrm{Fe} / \mathrm{H}]<-2$.

The lines selected in this work are given in Table 1 . The transition probabilities were taken from different experimental and theoretical sources. According to NIST, the $\operatorname{Sr}$ I $g f$-values are very accurate, the uncertainty is lower than 1 percent for the $4607 \AA$ line and better than 10 percent for the $7070 \AA$ 
Table 1. Lines of Sr I and Sr II selected for abundance calculations.

\begin{tabular}{|c|c|c|c|c|c|c|c|}
\hline $\begin{array}{l}\lambda \\
\AA \\
\end{array}$ & $\begin{array}{r}E_{\text {low }} \\
{[\mathrm{eV}]}\end{array}$ & Low & $\begin{array}{c}E_{\text {up }} \\
{[\mathrm{eV}]}\end{array}$ & Up & $\log g f$ & 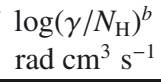 & $\log C_{6}{ }^{c}$ \\
\hline \multicolumn{8}{|l|}{ Sr I } \\
\hline 4607.33 & 0.00 & $5 s^{2}{ }^{1} S_{0}$ & 2.69 & $5 p^{1} \mathrm{P}_{1}^{\circ}$ & 0.283 & -7.53 & -31.2 \\
\hline 7070.01 & 1.85 & $5 \mathrm{p}^{3} \mathrm{P}_{2}^{\circ}$ & 3.60 & $6 s^{3} s_{1}^{1}$ & -0.020 & -7.15 & -30.2 \\
\hline \multicolumn{8}{|l|}{ Sr II } \\
\hline $4077.71^{a}$ & 0.00 & $5 \mathrm{~s}^{2} \mathrm{~S}_{1 / 2}$ & 3.04 & $5 p^{2} \mathrm{P}_{3 / 2}^{\circ}$ & 0.158 & -7.81 & -32.0 \\
\hline 4167.80 & 2.94 & $5 \mathrm{p}^{2} \mathrm{P}_{1 / 2}^{\mathrm{o}}$ & 5.92 & $6 s^{2} S_{1 / 2}^{3 / 2}$ & -0.502 & -7.81 & -32.0 \\
\hline $4215.52^{a}$ & 0.00 & $5 \mathrm{~s}^{2} \mathrm{~S}_{1 / 2}$ & 2.94 & $5 p^{2} \mathrm{P}_{1 / 2}^{\circ}$ & -0.155 & -7.81 & -32.0 \\
\hline 10036.65 & 1.81 & $4 \mathrm{~d}^{2} \mathrm{D}_{3 / 2}$ & 3.04 & $5 \mathrm{p}^{2} \mathrm{P}_{3 / 2}^{\mathrm{o}}$ & -1.194 & -7.64 & -31.5 \\
\hline 10327.31 & 1.84 & $4 d^{2} D_{5 / 2}^{j / 2}$ & 3.04 & $5 p^{2} \mathrm{P}_{3 / 2}^{\circ / 2}$ & -0.240 & -7.64 & -31.5 \\
\hline 10914.89 & 1.80 & $4 d^{2} D_{3 / 2}^{j / 2}$ & 2.94 & $5 p^{2} P_{1 / 2}^{\circ / 2}$ & -0.474 & -7.64 & -31.5 \\
\hline
\end{tabular}

Notes. ${ }^{(a)}$ blended. ${ }^{(b)}$ Log of the FWHM per H atom at 10000 K. ${ }^{(c)} C_{6}$ are in the units of $\mathrm{cm}^{6} \mathrm{~s}^{-1}$.

subordinate line. These $g f$-values were taken from the laboratory analysis of Parkinson (1976) and García \& Campos (1988), respectively. The oscillator strengths of the near-IR Sr II lines were adopted from the only available laboratory results of Gallagher (1967). They are fully consistent with the most recent ab initio calculations of Bautista et al. (2002), which were also adopted in the NLTE model atom.

Damping widths for the calculation of broadening due to elastic collisions with $\mathrm{HI}$ are available for the Sr II lines from the quantum-mechanical calculations of Barklem et al. (2000), and for the SrI lines they were kindly provided by the referee. We adopted the values from Barklem et al. for the near-IR Sr II transitions. However, for the strong resonance Sr II lines, which are very sensitive to this parameter, our spectrum synthesis calculations indicate values lower by $\sim 20$ percent. The question is whether this difference can be attributed to the uncertainties of the theoretical data arising because of certain approximations in the Anstee, Barklem, and O'Mara (ABO) theory. These include the representation of the interaction potential and collisional dynamics and have been investigated by Kerkeni et al. (2004) for neutral atoms of $\mathrm{Mg}, \mathrm{Sr}, \mathrm{Ca}$, and $\mathrm{Na}$. These authors found that while the semi-classical description of a collision is a sufficiently good approximation, the ABO potentials become quite inaccurate at small interatomic separations. However, the effect of the latter is rather to underestimate the line width, which clearly does not explain our finding. For the Sr II 4167 line we assumed the same $\gamma$ as for the resonance lines, but note that the line is too weak even in the solar spectrum to be sensitive to this parameter at all.

The adopted values of damping widths at $10000 \mathrm{~K}$ per $\mathrm{H}$ atom, $\gamma / N_{\mathrm{H}}$, as well as the commonly used interaction constants in the van der Waals-type interaction $\mathrm{C}_{6}$ computed for $T=5780 \mathrm{~K}$ are given in Table 1 . Here, the latter is a parameter in the Unsöld (1955, Eqs. (82), (48)) formula needed to reproduce the correct line width $\gamma$ at the given temperature ${ }^{5}$.

For the 4077 SrII line, which will be used for the abundance determinations in Sect. 4, we included the hyperfine structure and isotopic shifts. The magnetic dipole and electric quadrupole constants were taken from Buchinger et al. (1990):

\footnotetext{
5 Note that $\mathrm{C}_{6}$ is often computed using the Unsöld (1955, Eqs. (82), (55)) approximation, in which the interaction constant derives from the effective principal quantum number of lower and upper levels of a transition. However, this approximation is known to yield too small damping constants (e.g. Barklem 2007, and references therein).
}

Table 2. Hyperfine structure and isotopic shift for the $4077 \AA$ A line.

\begin{tabular}{lcc}
\hline \hline Isotope & $\begin{array}{c}\lambda \\
\AA\end{array}$ & $\log g f$ \\
\hline 87 & 4077.697 & -1.645 \\
87 & 4077.699 & -1.485 \\
84 & 4077.708 & -2.094 \\
86 & 4077.709 & -0.848 \\
88 & 4077.710 & 0.075 \\
87 & 4077.724 & -1.465 \\
87 & 4077.725 & -1.956 \\
\hline
\end{tabular}

$A\left(5 \mathrm{~s}^{2}{ }^{1} \mathrm{~S}_{0}\right)=-1000.5 \mathrm{MHz}, A\left(5 \mathrm{p}^{2} \mathrm{P}_{3 / 2}^{\circ}\right)=-36 \mathrm{MHz}, B$ $\left(5 \mathrm{p}^{2} \mathrm{P}_{3 / 2}^{\circ}\right)=88.5 \mathrm{MHz}$. The data agree with the older experiment by Borghs et al. (1983) and theoretical calculations by $\mathrm{Yu}$ et al. (2004). Isotope shifts for ${ }^{84} \mathrm{Sr},{ }^{86} \mathrm{Sr},{ }^{87} \mathrm{Sr}$ were taken from Borghs et al. (1983) and the solar isotopic ratios were adopted ${ }^{6}$. The wavelengths and $\log g f$ values of the hyperfine structure (HFS) components are given in Table 2 . We note that the $4215 \AA$ Sr I line was only used for a comparative LTE to NLTE analysis for the reasons discussed in Sect. 3.3.

\section{Statistical equilibrium of $\mathrm{Sr}$}

The departure coefficients ${ }^{7}$ for selected levels of $\mathrm{Sr}$ are shown as a function of continuum optical depth $\log \tau_{\mathrm{c}}$ at 500 in Fig. 4 for the model atmospheres with parameters corresponding to dwarfs and giants with $[\mathrm{Fe} / \mathrm{H}]=0$ and -2.4 . Only the levels that give rise to the spectral lines selected for the abundance analysis (Table 1) are included in the plots. These are two levels of Sr I, forming the resonance lines at $4607 \AA$, and two levels of Sr II, which are connected by the $4077 \AA$ transition commonly used in the analysis of metal-poor stars.

\subsection{NLTE effects in Srl}

Sr I with the ionization potential $5.69 \mathrm{eV}$ is a trace atom in the the atmospheres of late-type stars. The ratio of $\mathrm{Sr}$ I/Sr II falls below $10^{-3}$ above continuum optical depth unity, and the departures from LTE in the distribution of atomic level populations are almost entirely caused by overionization. The behavior of the SrI departure coefficients with stellar parameters therefore resembles that of similar trace atoms, such as Co I (Bergemann et al. 2010) or Cr I (Bergemann \& Cescutti 2010). The levels are underpopulated and their $b_{i}$-factors monotonously decrease outward. The ground state $5 \mathrm{~s}^{2}{ }^{1} \mathrm{~S}$ with the ionization edge at $2177 \AA$ is also strongly overionized and decouples from the other lowexcited levels because collisions cannot bridge the large energy gap of $\sim 2 \mathrm{eV}$ between them. Line transitions have a small influence on the statistical equilibrium of Sr I, causing some changes of the level populations only in the solar-metallicity models. For example, in the metal-rich models (Fig. 4, left panel), the departure coefficient of the $5 \mathrm{p}^{1} \mathrm{P}^{\mathrm{o}}$ level drops below that of the $5 \mathrm{~s}^{2}{ }^{1} \mathrm{~S}$ at $\log \tau_{\mathrm{c}} \approx-1$, marking the depth at which the 4607 resonance line becomes optically thin, and spontaneous transitions depopulate the upper level of the transition.

In the metal-poor models (Fig. 4, right panel), the coupling of the levels by radiative bound-bound transitions and collisions

\footnotetext{
6 http://wWw.nist.gov/pml/data/comp.cfm

7 The departure coefficients are defined as the ratio of NLTE to LTE level number densities, $b_{i}=n_{i}^{\mathrm{NLTE}} / n_{i}^{\mathrm{LTE}}$.
} 
M. Bergemann et al.: Statistical equilibrium of Sr in late-type stars
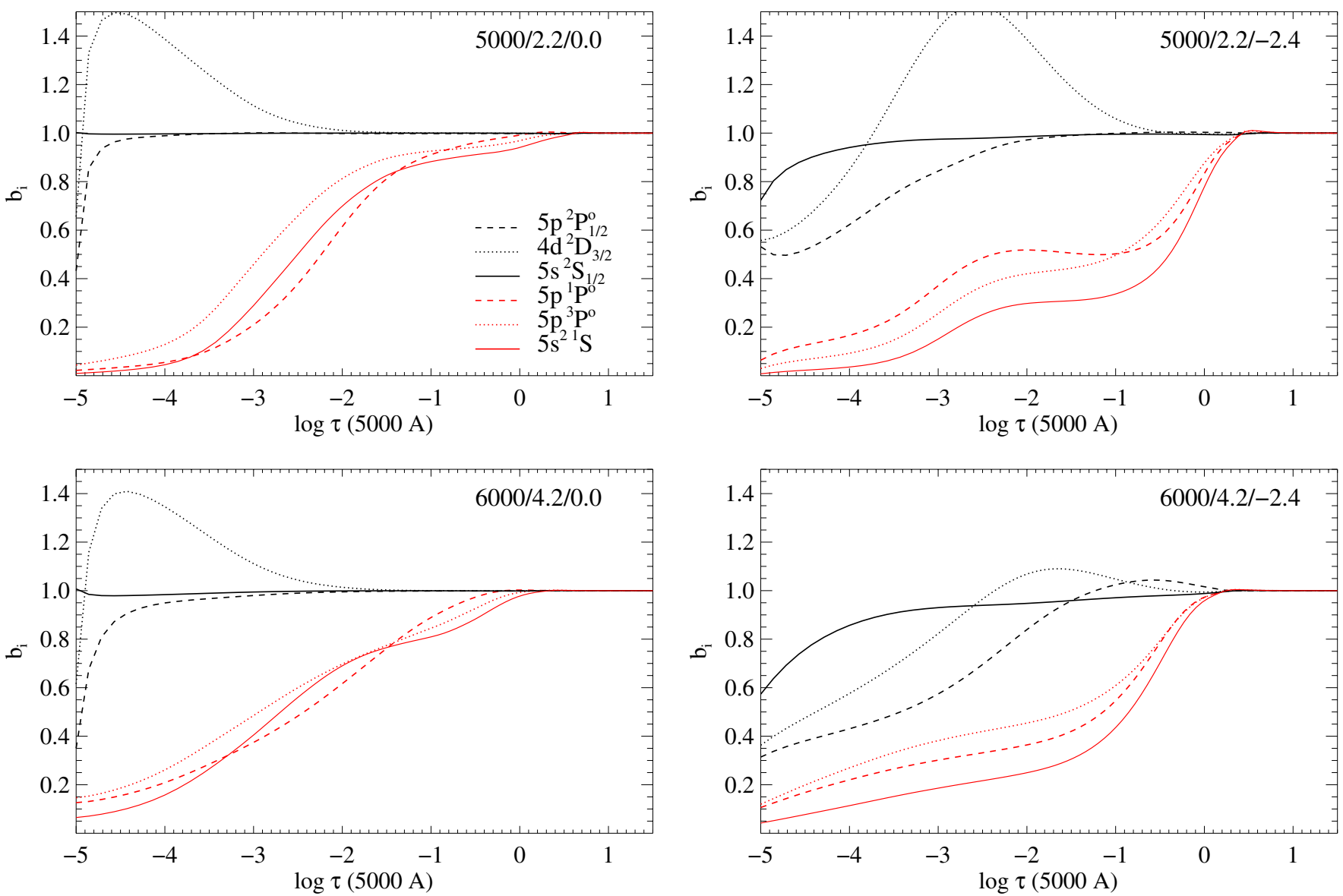

Fig. 4. Departure coefficients $b_{i}$ of selected $\mathrm{Sr}$ I and $\mathrm{Sr}$ II levels for different stellar parameters, $T_{\text {eff }}, \log g$, and $[\mathrm{Fe} / \mathrm{H}]$ (indicated in each sub-plot). Red: Sr I levels, $5 \mathrm{~s}^{2}{ }^{1} \mathrm{~S}$ (solid), $5 \mathrm{p}{ }^{3} \mathrm{P}^{\circ}$ (dotted), $5 \mathrm{p}^{1} \mathrm{P}^{\circ}$ (dashed). Black: Sr II levels, $5 \mathrm{~s}^{2} \mathrm{~S}_{1 / 2}$ (solid), $4 \mathrm{~d}^{2} \mathrm{D}_{3 / 2}$ (dotted), $5 \mathrm{p}^{2} \mathrm{P}_{1 / 2}^{\circ}$ (dashed).

diminishes ${ }^{8}$ even though the collision rates between the lowexcited levels are high enough to keep them in detailed balance even in the models with $[\mathrm{Fe} / \mathrm{H}]=-1$. The populations are now set primarily by the balance between radiative ionizations and recombinations, and the run of $b_{i}$-factors with $\log \tau_{\mathrm{c}}$ simply reflects the mean intensity vs. Planck function inequalities, $J_{v} \neq B_{v}$, at the frequencies of the level photoionization continua at each depth point. For example, in the model with $T_{\text {eff }}=6000 \mathrm{~K}, \log g=4.2$, and $[\mathrm{Fe} / \mathrm{H}]=-2.4$, the lowest energy levels $5 \mathrm{p}{ }^{3} \mathrm{P}^{\mathrm{o}}$ and $5 \mathrm{p}{ }^{1} \mathrm{P}^{\mathrm{o}}$ decouple from each other and from the continuum at $\log \tau_{\mathrm{c}} \approx 0$. Then, in a very narrow interval of optical depths, $-1<\log \tau_{\mathrm{c}}<0$, their departure coefficients demonstrate a sudden drop following the steep temperature gradient of the atmosphere, and thus the rapid increase of $J_{v}-B_{v}$ imbalance (note that matter becomes transparent to radiation above ionization edges of both levels, 3202 and $4126 \AA$ at $\log \tau_{\mathrm{c}}=+0.11$ and $\log \tau_{\mathrm{c}}=+0.05$, respectively). Farther out, $\log \tau_{\mathrm{c}}<-1$, where the mean intensity as well as the local kinetic temperature remain roughly constant, the departure coefficients smooth out, slowly decreasing outward.

The NLTE effects on the formation of the Sr I line at $4607 \AA$ are generally to decrease the line opacity, shifting the line $\tau_{v}$ scale to the deeper hotter atmospheric layers and, thus, leading to weaker lines compared to the LTE case.

\footnotetext{
8 Note that we did not include the H I collisions as discussed in Sect. 4.
}

\subsection{NLTE effects in Srll}

The NLTE effects on the Sr II levels are caused by the nonequilibrium excitation in the line transitions. This was extensively investigated by Belyakova \& Mashonkina (1997) and Short \& Hauschildt (2006), and our results are qualitatively very similar to these studies. The analysis of radiative and collisional rates populating and de-populating the levels, as well as trial calculations with atomic models devoid of some key transitions, in particular the resonance and subordinate lines, allows us to draw the following conclusions.

In the solar-metallicity models (Fig. 4, left panel), the ground state, $5 \mathrm{~s}{ }^{2} \mathrm{~S}\left(\lambda_{\mathrm{thr}} \sim 1130 \AA\right.$ A $)$ and the lowest odd level $5 \mathrm{p}^{2} \mathrm{P}^{\circ}$ level $\left(\lambda_{\text {thr }} \sim 1540 \AA, E \sim 3 \mathrm{eV}\right)$ have LTE populations out to $\log \tau_{\mathrm{c}} \sim-3$, and become underpopulated only in the outermost layers, which are transparent to the radiation across their ionization edges. Still, there is some low net radiative excitation in the transitions from these levels upward, which very efficiently populates the levels with excitation energy $E \geq 6 \mathrm{eV}$, such as $5 \mathrm{~d}^{2} \mathrm{D}, 6 \mathrm{p}^{2} \mathrm{P}^{\circ}$, and $7 \mathrm{~s}^{2} \mathrm{~S}$ (not shown in the plots) due to their low Boltzmann factors. The highly-excited levels are, however, also overionized, which in turn increases the number density of the Sr III ground state. The lowest metastable Sr II level $4 \mathrm{~d}^{2} \mathrm{D}$ $(E \sim 1.8 \mathrm{eV})$ is nearly in thermal equilibrium with $5 \mathrm{p}^{2} \mathrm{P}^{\circ}$ because of the dominance of collision rates in the deeper layers but in the outer layers it gains appreciable overpopulation through the photon losses in the near-IR transitions between the two levels. Indeed, this process is a part of photon suction in a sequence 

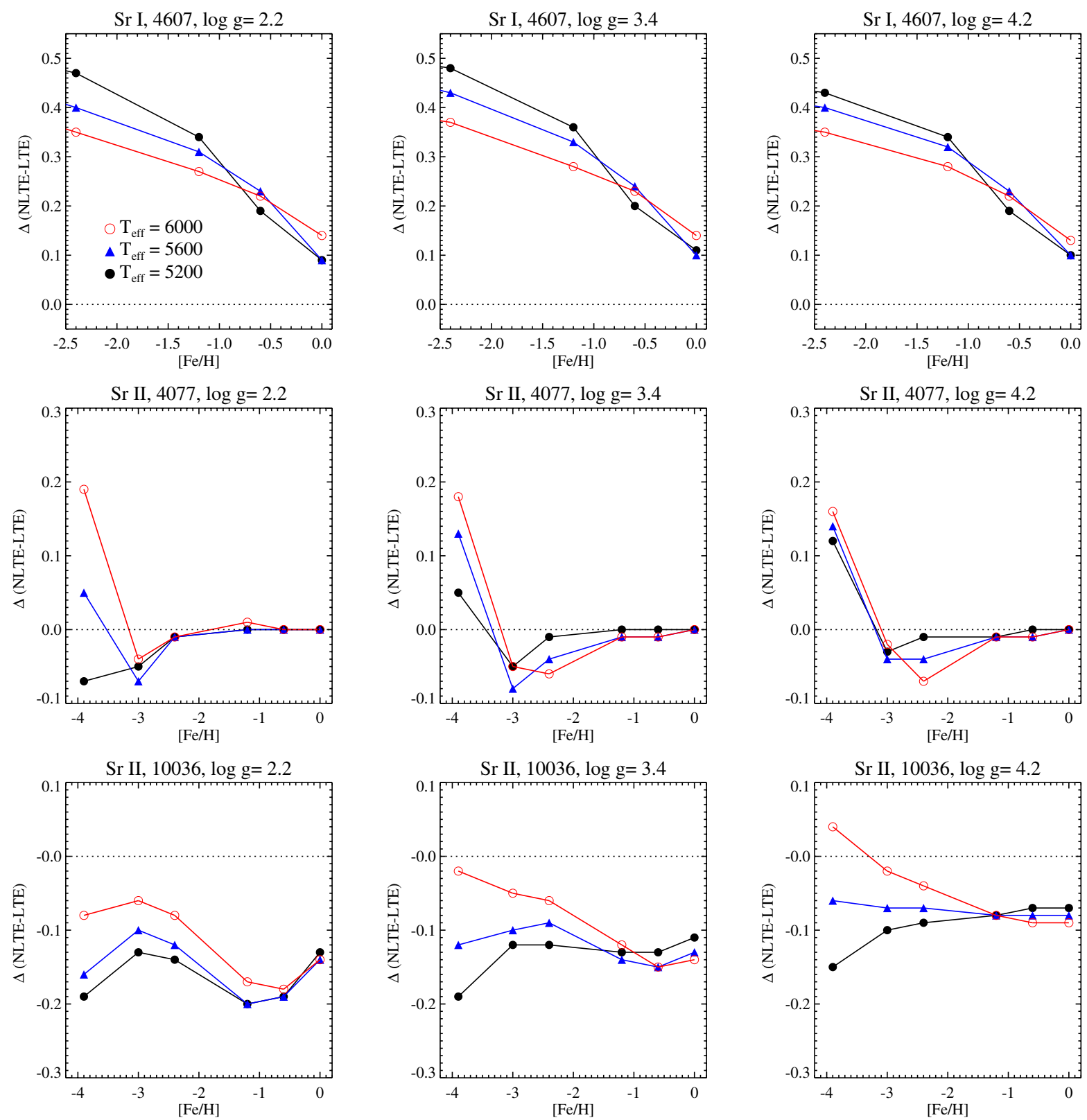

Fig. 5. NLTE abundance corrections for the Sr I and Sr II lines. Note the different $x$-axis scales.

of low-frequency transitions, which connect $4 \mathrm{~d}^{2} \mathrm{D}$ to many highlevels close to the continuum. Many of these transitions become optically thin at $\log \tau_{\mathrm{c}}<-3$, so that spontaneous de-excitation leads to the overpopulation $4 \mathrm{~d}^{2} \mathrm{D}$, the lowest energy state of the cascading sequence.

The departures from LTE change with decreasing metallicity (Fig. 4, right panel). First, the line transitions weaken and and overpopulation of $4 \mathrm{~d}^{2} \mathrm{D}$ occurs in the deeper layers compared to the solar metallicity models. Also, radiative pumping in the resonance transitions at 4077 and $4215 \AA$ is more efficient because of the larger radiative fluxes. This process leads to a marked overpopulation of the $5 \mathrm{p}^{2} \mathrm{P}^{\circ}$ level at $-1<\log \tau_{\mathrm{c}}<0$, especially in the hotter model, $T_{\text {eff }}=6200 \mathrm{~K}$. Beyond these optical depths, overionization dominates and all low-lying levels become underpopulated. The importance of strong line scattering was tested by excluding the resonance lines and subordinate lines at $1 \mu \mathrm{m}$ from the model atom. The overpopulation of the $5 \mathrm{p}^{2} \mathrm{P}^{\circ}$ and $4 \mathrm{~d}^{2} \mathrm{D}$ vanished for the metal-rich and metal-poor models.

\subsection{NLTE abundance corrections}

To quantify the effect of NLTE on the abundance determinations, we used the concept of the NLTE abundance correction $\Delta_{\text {NLTE}}$, where

$\Delta_{\mathrm{NLTE}}=\log \epsilon(\mathrm{Sr})_{\mathrm{NLTE}}-\log \epsilon(\mathrm{Sr})_{\mathrm{LTE}}$

is the the logarithmic correction that has to be applied to an LTE abundance determination of a specific line to obtain the exact value corresponding to the use of NLTE line formation. These values were computed for a grid of model atmospheres by equalizing the NLTE equivalent widths though varying the element abundance in the LTE calculations. The NLTE abundance corrections for the 4077, 4607 and 10036 line are given in Tables 35 for a wide range of stellar parameters. Fig. 5 (top panel) also shows the values of $\Delta_{\text {NLTE }}$ for selected model atmospheres as a function of effective temperature, gravity, and $[\mathrm{Fe} / \mathrm{H}]$. 
As evident from Fig. 5 (top panel) and Table 3, the NLTE abundance corrections for the $4607 \AA$ are always positive, and they are maximum for cool giants with subsolar metallicity, $[\mathrm{Fe} / \mathrm{H}]<-1$. This behavior can be easily understood by inspecting the plots of the departure coefficients (Fig. 4, top right panel): at the typical depths of the resonance line formation, $\log \tau_{\mathrm{c}} \sim 0 \ldots-0.5$, the level populations are already depleted by a factor of two compared to LTE. In the models of warmer metal-poor dwarfs, the line formation is confined to the deeper layers, $\log \tau_{\mathrm{c}} \sim 0$, where the NLTE effects in the Sr I ionization balance are weaker. This is reflected in the behavior of the abundance corrections, which also decrease. $\Delta_{\text {NLTE }}$ are comparatively small for the warmest models, corresponding to horizontal branch stars $\left(T_{\text {eff }}=6200\right.$ and $\left.\log g=2.2\right)$. Note, however, that at $T_{\text {eff }}>6000 \mathrm{~K}$ and $[\mathrm{Fe} / \mathrm{H}]<-1.5$ the $4607 \AA$ line is very weak, $W_{\lambda}<0.5 \mathrm{m \AA}$, and, thus, is not useful for abundance determinations. In contrast, the line could be suitable for the analysis in sufficiently good-quality $(R>20000, S / N>50)$ spectra of giants and subgiants down to $[\mathrm{Fe} / \mathrm{H}] \sim-2.5$.

As described in the previous section, the formation of Sr II lines is also significantly affected by the NLTE effects. Inspection of Table 4 and Fig. 5 (middle panel) shows that for the resonance Sr II line at $4077 \AA$ the NLTE abundance corrections are typically negative for stars with metallicity $[\mathrm{Fe} / \mathrm{H}]>-3$, but rapidly increase for more metal-poor stars with $T_{\text {eff }}>5000 \mathrm{~K}$ and/or $\log g>3$. The amplitude of $\Delta_{\mathrm{NLTE}}$ increases with $T_{\mathrm{eff}}$ and decreasing $\log g$. Another resonance Sr II line at $4215 \AA$ shows the same pattern and the NLTE abundance corrections are nearly identical; we caution, however, that this line is blended by an Fe I line and should be avoided in abundance analyses of cool stars. The implication is that, compared to NLTE, traditional LTE studies that rely on the resonance Sr II lines underestimate the abundance of $\mathrm{Sr}$ in very metal-poor stars, but somewhat overestimate it for less metal-poor objects, such as those of thick and thin disk stars of the Galaxy.

For the near-IR subordinate Sr II lines, the NLTE abundance corrections are typically negative (Table 5 and Fig. 5, bottom panel), being as large as -0.2 dex even for mildly metal-poor giants; this behavior is consistent with the discussion in Sect. 3.2, because the NLTE effects are caused by strong line scattering. However, $\Delta_{\text {NLTE }}$ are usually within -0.1 dex for dwarfs and become mildly positive only at very low metallicity, $[\mathrm{Fe} / \mathrm{H}]<-3$ and $T_{\text {eff }}>6000 \mathrm{~K}$. The NLTE abundance corrections presented in Table 5 are quite similar for the other two members of the near-IR triplet, i.e., the lines at 10327 and $10914 \AA$, and can be provided on request.

Our NLTE abundance corrections are in line with the results of Short \& Hauschildt (2006), although these authors investigated the formation of the resonance SrII lines only. They found NLTE line strengthening for the giant models with $[\mathrm{Fe} / \mathrm{H}]=-1 \ldots-2$ and NLTE weakening for the models with $[\mathrm{Fe} / \mathrm{H}]=-4 \ldots-5$. However, we note some qualitative differences with Andrievsky et al. (2011). For example, for the nearIR Sr II lines their abundance corrections at $[\mathrm{Fe} / \mathrm{H}]=-3$ and $T_{\text {eff }}=4800-5300 \mathrm{~K}$ are between -0.3 to $-0.5 \mathrm{dex}$ and are very sensitive to surface gravity (their Fig. 7, top panel). In contrast, our values for the $10036 \AA$ line remain within $\sim-0.1 \mathrm{dex}$ for any $\log g$ value in this $[\mathrm{Fe} / \mathrm{H}]$ and $T_{\text {eff }}$ range. For the resonance Sr II line at $4077 \AA$, our $\Delta_{\text {NLTE }}$ are mildy negative for any $\log g$ and $T_{\text {eff }}$ at $[\mathrm{Fe} / \mathrm{H}]=-3$, whereas Andrievsky et al. (2011) obtain large positive corrections for dwarfs and negative $\Delta_{\text {NLTE }}$ for giants. The differences are most likely caused by the differences in the NLTE model atoms, as described in Sect. 2.2.
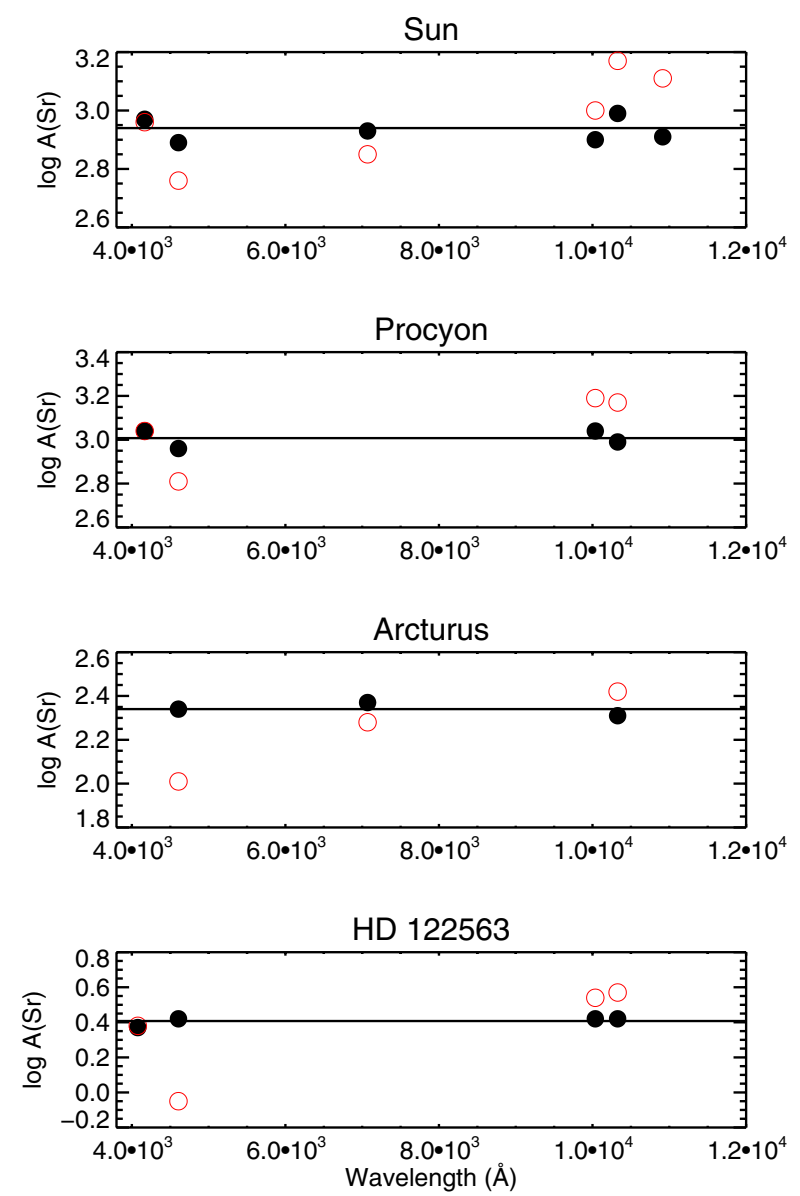

Fig. 6. NLTE and LTE line-by-line Sr abundances for the selected stars. In contrast to LTE (red open circles), the SrI and Sr II lines provide consistent abundances in NLTE (black filled circles).

\section{Application to the abundance analysis of late-type stars}

In addition to the Sun, three other late-type stars were selected for the abundance analysis. The principle selection criteria were that stellar parameters are well-constrained by independent techniques (interferometry, parallaxes), observations cover a large wavelength interval, and their quality is very high so that the 4607 Sr I line and the near-IR Sr II triplet can be accurately measured. Stellar parameters for the warm turn-off star Procyon, the moderately metal-poor giant Arcturus (HD 124897), and the very metal-poor giant HD 122563 were taken from Korn et al. (2003), Tsuji (2009), and Mashonkina et al. (2008), respectively (Table 6). The accuracy of these parameters was verified in our recent analysis of Fe NLTE statistical equilibrium with mean 3D model atmospheres (Bergemann et al. 2012). We stress that it is not our goal here to obtain $\mathrm{Sr}$ abundances for a large sample of stars, which will be the focus of our forthcoming publications (Hansen et al., in prep.; Ruchti et al., in prep.), but rather to test the performance of the new NLTE model atom.

For the spectrum synthesis (Sect. 2.1), we used the solar KPNO flux spectrum (Kurucz et al. 1984). The spectra of Arcturus (HD 124897), Procyon (HD 61421), and HD 122563 were retrieved from the UVESPOP database (Bagnulo et al. 2003). These UVES (VLT, Paranal) spectra have a slitdetermined resolution of $\sim 80000$ and a signal-to-noise ratio $S / N \sim 300$ near $5000 \AA$. 

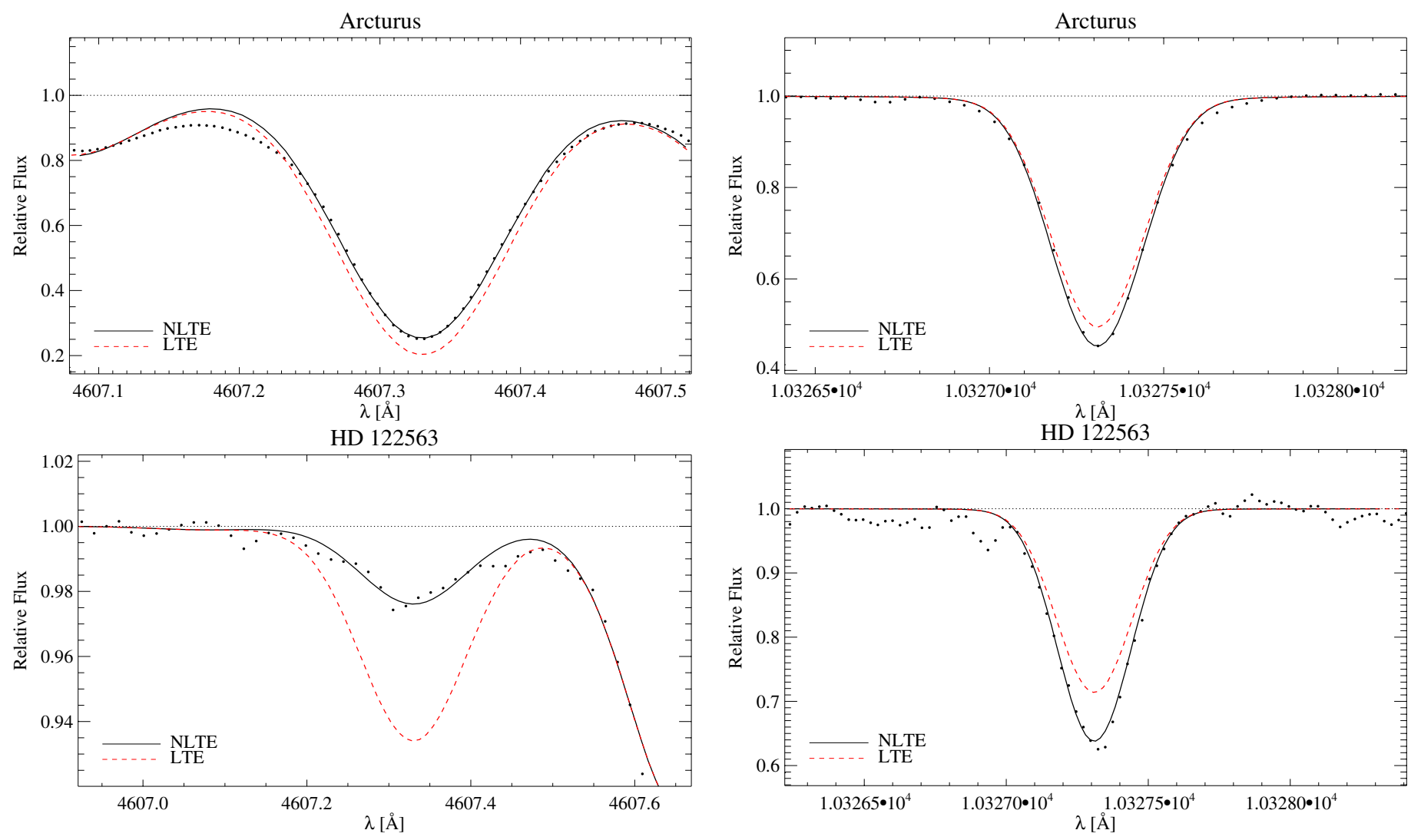

Fig. 7. NLTE and LTE line profiles of the Sr I and Sr II lines for selected stars compared with observed stellar spectra.

Table 6. Basic parameters and the Sr abundances for the selected stars.

\begin{tabular}{lccccccrr}
\hline \hline \multirow{2}{*}{ Star } & $\begin{array}{c}T_{\mathrm{eff}} \\
\mathrm{K}\end{array}$ & $\begin{array}{l}\log g \\
(\mathrm{cgs})\end{array}$ & {$[\mathrm{Fe} / \mathrm{H}]$} & $\begin{array}{c}\xi \\
\mathrm{km} \mathrm{s}^{-1}\end{array}$ & {$[\mathrm{Sr} / \mathrm{Fe}]$} & {$[\mathrm{Sr} \mathrm{II} / \mathrm{Fe}]$} & {$[\mathrm{Sr} / / \mathrm{Fe}]$} & {$[\mathrm{Sr} \mathrm{II} / \mathrm{Fe}]$} \\
\hline HD 61421 & 6510 & 3.96 & -0.03 & 1.8 & -0.08 & 0.27 & 0.07 & 0.15 \\
HD 122563 & 4600 & 1.60 & -2.50 & 1.8 & -0.47 & 0.08 & 0.00 & -0.02 \\
HD 124897 & 4300 & 1.50 & -0.50 & 1.5 & -0.28 & 0.00 & -0.07 & -0.11 \\
\hline
\end{tabular}

The solar abundance was determined using five $\mathrm{Sr}$ lines from Table 1, adopting $T_{\text {eff }}=5777, \log g=4.44,[\mathrm{Fe} / \mathrm{H}]=$ 0 , and $\xi_{\mathrm{t}}=1 \mathrm{~km} \mathrm{~s}^{-1}$. The results are largely discrepant in LTE: $\log \epsilon_{\text {SrI }}=2.81 \pm 0.06$ and $\log \epsilon_{\text {SrII }}=3.06 \pm 0.1$. The NLTE model atom successfully eliminates this problem. The abundances from the two ionization stages are fully consistent $\log \epsilon_{\text {SrI }}=2.91 \pm 0.03$ and $\log \epsilon_{\text {SrII }}=2.94 \pm 0.04$ in NLTE. Here, the error corresponds to one standard deviation of the line sample. The NLTE result also agrees with the meteoritic abundance of $\mathrm{Sr}, \log \epsilon=2.90 \pm 0.03$ dex (Lodders et al. 2009). We note that the meteoritic abundance of Sr given by Asplund et al. (2009), Sr, $\log \epsilon=2.88 \pm 0.03 \mathrm{dex}$, is slightly lower, which is a consequence of different reference $\mathrm{Si}$ abundances. The solar $\mathrm{Sr}$ abundance was also determined by Barklem \& O'Mara (2000), Andrievsky et al. (2011), and Mashonkina \& Gehren (2001). The last two references performed NLTE calculations that generally agree with our results, both in terms of NLTE abundance corrections and absolute abundances. We caution, however, that this agreement is not particularly meaningful, because other parameters in the modeling are different. Mashonkina \& Gehren (2001) adopted $\xi_{\mathrm{t}}=0.8 \mathrm{~km} \mathrm{~s}^{-1}$ and employed the older (ODF) version of the MAFAGS model atmospheres. Barklem \& O'Mara (2000) recovered meteoritic Sr abundance from the neutral $\mathrm{Sr}$ lines even in LTE, which is not unexpected because they used $\xi_{\mathrm{t}}=0.85 \mathrm{~km} \mathrm{~s}^{-1}$ and the solar Holweger-Müller (1974) model atmosphere ${ }^{9}$.

The LTE and NLTE Sr abundances determined for the reference stars are given in Table 6 and a line-by-line comparison is shown in Fig. 6. Several examples of synthetic and observed line profiles are shown in Fig. 7. Comparison of the $[\mathrm{Sr} \mathrm{I} / \mathrm{Fe}]$ and [Sr II $/ \mathrm{Fe}]$ from Table 6 reveals that the LTE approach predicts a systematic discrepancy between the lines of the two ionization stages that is also evident from the Fig. 6. The difference between the Sr I and the near-IR Sr II lines, which are almost insensitive to damping, is about 0.3 to 0.5 dex. This is far beyond the uncertainties in stellar parameters, which are $\sim 80 \mathrm{~K}$ for $T_{\text {eff }}$, $0.1 \mathrm{dex}$ for $\log g$, and $0.1 \mathrm{dex}$ for $[\mathrm{Fe} / \mathrm{H}]$. Also, the uncertainties of the transition probabilities are far smaller than that (Sect. 2.3).

9 The semi-empirical Holweger-Müller model atmosphere is hotter than all solar theoretical models by $\sim 150 \mathrm{~K}$ over the line formation layers and, in this way, it "mimics" NLTE effects for some atoms. 
M. Bergemann et al.: Statistical equilibrium of $\mathrm{Sr}$ in late-type stars

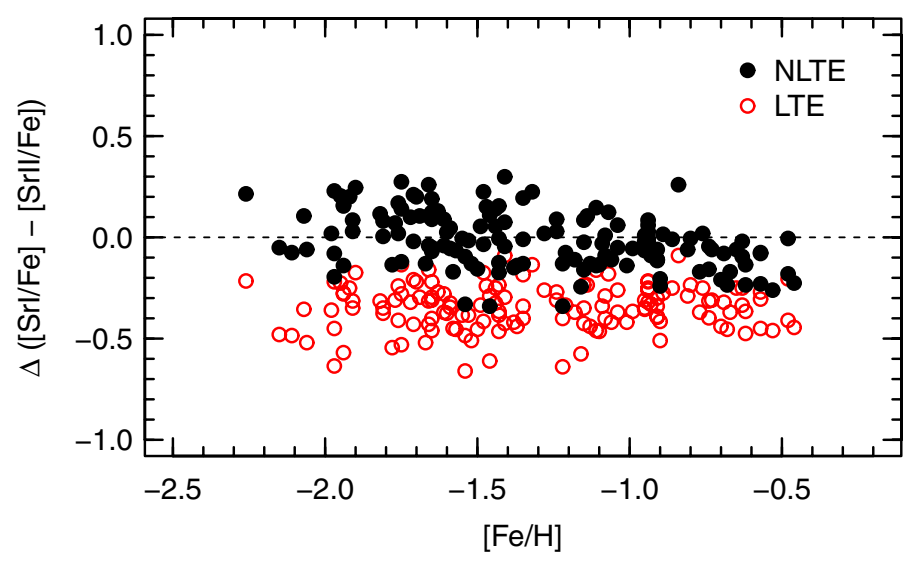

Fig. 8. NLTE and LTE abundance differences between Sr I and Sr II lines for the representative sample of thick-disk and halo stars from Ruchti et al. (2011).

The NLTE model atom in contrast provides realistic ionization balance, which significantly reduces the scatter between the $\mathrm{Sr}$ lines of different ionization stages and excitation energies (Fig. 6). In this respect, a good agreement between the NLTE abundances from the near-IR SrII and visual resonance Sr I lines is especially important, because these are very sensitive to NLTE and the effects are of different nature, i.e., overionization-stipulated decrease of opacity in the 4607 line and source function depletion in the lines of the $1 \mu \mathrm{m}$ triplet.

The analysis of a larger sample of stars supports the importance of NLTE effects on the ionization balance of Sr. This is evident from Fig. 8, which shows the difference between the mean abundances computed using the Sr I and Sr II lines for a sample of thick-disk and halo RAVE stars with high-resolution spectroscopic follow-up observations (see Ruchti et al. 2011). In LTE, the mean offset between the two ionization stages is $\sim 0.4$ dex and it is alleviated when the NLTE effects are taken into account. A detailed analysis of the data will be presented in Ruchti et al. (in prep.).

We therefore conclude that NLTE must be taken into account in abundance analysis of resonance SrI and subordinate $\mathrm{Sr}$ II lines in spectra of late-type stars. The resonance Sr II lines are also sensitive to NLTE effects at low metallicities, and abundances determined in LTE are largely underestimated. It is also important to note that the NLTE effects for Sr might be stronger than our estimates, because of interlocking with NLTE-affected lines of other atoms, as demonstrated by Short \& Hauschildt (2006).

\section{Conclusions}

We investigated the statistical equilibrium of $\mathrm{Sr}$ in the atmospheres of late-type stars. The NLTE model atom was constructed using new atomic data, computed with the R-matrix method, which includes levels, transition probabilities, photoionization, and electron-impact excitation cross-sections.

The NLTE effects are significant for the $\mathrm{Sr}$ I resonance line at $4607 \AA$ and are stipulated by overionization. For the resonance Sr II lines, which are typically observed in metal-poor FGK stars, our model predicts small deviations from LTE, although NLTE effects become important at very low metallicity, $[\mathrm{Fe} / \mathrm{H}]<-3$, reaching +0.2 dex for very metal-poor turnoff stars. The near-IR Sr II triplet shows substantial sensitivity to NLTE, and the NLTE corrections vary between +0.1 and -0.2 dex depending on the model metallicity, temperature, and surface gravity.

The NLTE model atom was applied to the analysis of the Sun and three stars with well-constrained stellar parameters. In contrast to the LTE approach, the NLTE model recovers ionization and excitation equilibria of $\mathrm{Sr}$ for all these reference stars, thus confirming that the NLTE modeling approach developed in this work provides a solid base for future abundance determinations of late-type stars.

The NLTE abundance corrections were provided for the important diagnostic SrI and Sr II lines for a grid of model atmospheres in the following range of stellar parameters $4400 \leq$ $T_{\text {eff }} \leq 6000 \mathrm{~K}, 2.2 \leq \log g \leq 4.6,-4 \leq[\mathrm{Fe} / \mathrm{H}] \leq 0$; for other $\mathrm{Sr}$ lines and other models the abundance corrections can be also computed on request.

Acknowledgements. Based on observations made with the European Southern Observatory telescopes (obtained from the ESO/ST-ECF Science Archive Facility). We thank Luca Sbordone for help with the revision of SIU. We are grateful to the referee Paul Barklem for providing some atomic data and very useful comments that helped to improve the paper.

\section{References}

Allen, C. W. 1973 (London: University of London, Athlone Press), 3rd edn. Andrievsky, S. M., Spite, F., Korotin, S. A., et al. 2011, A\&A, 530, A105 Anstee, S. D., \& O'Mara, B. J. 1995, MNRAS, 276, 859

Asplund, M., Grevesse, N., Sauval, A. J., \& Scott, P. 2009, ARA\&A, 47, 481 Badnell, N. R. 1986, J. Phys. B: Atom. Mol. Phys., 19, 3827

Badnell, N. R. 1997, J. Phys. B: Atom. Mol. Phys., 30, 1

Bagnulo, S., Jehin, E., Ledoux, C., et al. 2003, The Messenger, 114, 10

Barklem, P. S. 2007, Spectral Line Shapes in Astrophysics, 938, 111

Barklem, P. S., \& O'Mara, B. J. 2000, MNRAS, 311, 535

Barklem, P. S., Piskunov, N., \& O'Mara, B. J. 2000, A\&AS, 142, 467

Barklem, P. S., Belyaev, A. K., Spielfiedel, A., Guitou, M., \& Feautrier, N. 2012, A\&A, 541, A80

Bautista, M. A., Gull, T. R., Ishibashi, K., Hartman, H., \& Davidson, K. 2002, MNRAS, 331, 875

Belyaev, A. K., \& Barklem, P. S. 2003, Phys. Rev. A, 68, 062703

Belyakova, E. V., \& Mashonkina, L. I. 1997, AZh, 74, 601

Bergemann, M., \& Cescutti, G. 2010, A\&A, 522, A9

Bergemann, M., Pickering, J. C., \& Gehren, T. 2010, MNRAS, 401, 1334

Bergemann, M., Lind, K., Collet, R., Magic, Z., \& Asplund, M. 2012, MNRAS, submitted

Borghs, G., de Bisschop, P., van Hove, M., \& Silverans, R. E. 1983, Hyperfine Interactions, 15, 177

Buchinger, F., Ramsay, E. B., Arnold, E., et al. 1990, Phys. Rev. C, 42, 2754

Burke, P. G., Hibbert, A., \& Robb, W. D. 1971, J. Phys. B: Atom. Mol. Phys., 4, 153

Butler, K., \& Giddings, J. 1985, Newsletter on Analysis of Astronomical Spectra No. 9, University College London

Cowan, J. J., Sneden, C., Burles, S., et al. 2002, ApJ, 572, 861

Drawin, H.-W. 1968, Z. Phys., 211, 404

Drawin, H. W. 1969, Z. Phys., 225, 470

Eissner, W., \& Nussbaumer, H. 1969, J. Phys. B: Atom. Mol. Phys., 2, 1028

Farouqi, K., Kratz, K.-L., Pfeiffer, B., et al. 2010, ApJ, 712, 1359

García, G., \& Campos, J. 1988, J. Quant. Spec. Radiat. Transf., 39, 477

Gehren, T., Liang, Y. C., Shi, J. R., Zhang, H. W., \& Zhao, G. 2004, A\&A, 413 1045

Gehren, T., Shi, J. R., Zhang, H. W., Zhao, G., \& Korn, A. J. 2006, A\&A, 451, 1065

Gratton, R. G., \& Sneden, C. 1994, A\&A, 287, 927

Grupp, F. 2004a, A\&A, 420, 289

Grupp, F. 2004b, A\&A, 426, 309

Gustafsson, B., Edvardsson, B., Eriksson, K., et al. 2008, A\&A, 486, 951

Jehin, E., Magain, P., Neuforge, C., et al. 1999, A\&A, 341, 241

José, J., \& Iliadis, C. 2011, Rep. Prog. Phys., 74, 096901

Kerkeni, B., Barklem, P. S., Spielfiedel, A., \& Feautrier, N. 2004, J. Phys. B At. Mol. Phys., 37, 677

Korn, A. J., Shi, J., \& Gehren, T. 2003, A\&A, 407, 691

Kurucz, R., \& Bell, B. 1995, Atomic Line Data, Kurucz CD-ROM No. 23 (Cambridge, Mass.: Smithsonian Astrophysical Observatory)

Lambert, D. L. 1993, Phys. Scr. T, 47, 186 
Lindgård, A., \& Nielson, S. E. 1977, Atomic Data and Nuclear Data Tables, 19, 533

Lodders, K., Palme, H., \& Gail, H.-P. 2009, Landolt-Börnstein - Group VI Astronomy and Astrophysics Numerical Data and Functional Relationships in Science and Technology Volume, 44

Mashonkina, L., \& Gehren, T. 2001, A\&A, 376, 232

Mashonkina, L., Zhao, G., Gehren, T., et al. 2008, A\&A, 478, 529

Parkinson, J. H. 1976, Roy. Soc. London Philos. Trans. Ser. A, 281, 375

Ralchenko, Yu., Kramida, A. E., Reader, J., \& NIST ASD Team (2011), NIST Atomic Spectra Database (ver. 4.1.0), http://physics.nist.gov/ asd [2012, March 24]. National Institute of Standards and Technology, Gaithersburg, MD

Reetz, J. 1999, Ph.D. Thesis, LMU München

Ruchti, G. R., Fulbright, J. P., Wyse, R. F. G., et al. 2011, ApJ, 737, 9
Rybicki, G. B., \& Hummer, D. G. 1991, A\&A, 245, 171

Rybicki, G. B., \& Hummer, D. G. 1992, A\&A, 262, 209

Schatz, H., Aprahamian, A., Goerres, J., et al. 1998, Phys. Rep., 294, 167

Short, C. I., \& Hauschildt, P. H. 2006, ApJ, 641, 494

Steenbock, W., \& Holweger, H. 1984, A\&A, 130, 319

Surman, R., McLaughlin, G. C., Ruffert, M., Janka, H.-T., \& Hix, W. R. 2008, ApJ, 679, L117

Travaglio, C., Hillebrandt, W., Reinecke, M., \& Thielemann, F.-K. 2004, A\&A, 425,1029

Tsuji, T. 2009, A\&A, 504, 543

Unsöld, A. 1955 (Berlin: Springer), 2. Aufl.

van Regemorter, H. 1962, ApJ, 136, 906

Wanajo, S., Janka, H.-T., \& Müller, B. 2011, ApJ, 726, L15 
M. Bergemann et al.: Statistical equilibrium of $\mathrm{Sr}$ in late-type stars

Table 3. NLTE abundance corrections for the $4607 \mathrm{Sr}$ I line.

\begin{tabular}{lrrrrrrrr}
\hline \hline$T_{\text {eff }}$ & {$[\mathrm{Fe} / \mathrm{H}]$} & & \multicolumn{7}{c}{$\Delta_{\text {NLTE }}$} \\
$\log g$ & & 2.20 & 2.60 & 3.00 & 3.40 & 3.80 & 4.20 & 4.60 \\
\hline 4400 & -3.0 & 0.54 & 0.51 & 0.47 & - & - & - & - \\
4400 & -2.4 & 0.49 & 0.49 & 0.49 & - & - & - & - \\
4400 & -1.2 & 0.29 & 0.29 & 0.28 & - & - & - & - \\
4400 & -0.6 & 0.27 & 0.26 & 0.24 & - & - & - & - \\
4400 & 0.0 & 0.19 & 0.18 & 0.16 & - & - & - & - \\
& & & & & & & & \\
4800 & -3.0 & 0.55 & 0.55 & 0.51 & 0.49 & 0.45 & - & - \\
4800 & -2.4 & 0.50 & 0.51 & 0.51 & 0.49 & 0.46 & 0.43 & 0.36 \\
4800 & -1.2 & 0.32 & 0.33 & 0.33 & 0.33 & 0.32 & 0.30 & 0.28 \\
4800 & -0.6 & 0.17 & 0.17 & 0.18 & 0.18 & 0.17 & 0.16 & 0.14 \\
4800 & 0.0 & 0.19 & 0.18 & 0.18 & 0.17 & 0.15 & 0.13 & 0.10 \\
& & & & & & & & \\
5200 & -3.0 & 0.50 & 0.52 & 0.52 & 0.50 & 0.47 & 0.45 & 0.42 \\
5200 & -2.4 & 0.47 & 0.48 & 0.49 & 0.48 & 0.45 & 0.43 & 0.41 \\
5200 & -1.2 & 0.34 & 0.35 & 0.36 & 0.36 & 0.35 & 0.34 & 0.32 \\
5200 & -0.6 & 0.19 & 0.20 & 0.20 & 0.20 & 0.20 & 0.19 & 0.17 \\
5200 & 0.0 & 0.09 & 0.10 & 0.10 & 0.11 & 0.11 & 0.10 & 0.09 \\
& & & & & & & & \\
5600 & -3.0 & 0.44 & 0.45 & 0.46 & 0.46 & 0.45 & 0.42 & 0.40 \\
5600 & -2.4 & 0.40 & 0.42 & 0.43 & 0.43 & 0.43 & 0.40 & 0.38 \\
5600 & -1.2 & 0.31 & 0.32 & 0.33 & 0.33 & 0.33 & 0.32 & 0.31 \\
5600 & -0.6 & 0.23 & 0.24 & 0.24 & 0.24 & 0.24 & 0.23 & 0.21 \\
5600 & 0.0 & 0.09 & 0.10 & 0.10 & 0.10 & 0.11 & 0.10 & 0.09 \\
& & & & & & & & \\
6000 & -3.0 & 0.39 & 0.39 & 0.39 & 0.39 & 0.39 & 0.37 & 0.35 \\
6000 & -2.4 & 0.35 & 0.36 & 0.36 & 0.37 & 0.36 & 0.35 & 0.34 \\
6000 & -1.2 & 0.27 & 0.28 & 0.28 & 0.28 & 0.28 & 0.28 & 0.27 \\
6000 & -0.6 & 0.22 & 0.23 & 0.23 & 0.23 & 0.23 & 0.22 & 0.21 \\
6000 & 0.0 & 0.14 & 0.14 & 0.14 & 0.14 & 0.13 & 0.13 & 0.12 \\
\hline & & & & & & & & \\
& & & & & \\
& & & & & \\
& & & & & \\
500 &
\end{tabular}

Table 4. NLTE abundance corrections for the $4077 \mathrm{Sr}$ II line.

\begin{tabular}{lrrrrrrrr}
\hline \hline$T_{\text {eff }}$ & {$[\mathrm{Fe} / \mathrm{H}]$} & & & \multicolumn{7}{c}{$\Delta_{\text {NLTE }}$} \\
$\log g$ & & 2.20 & 2.60 & 3.00 & 3.40 & 3.80 & 4.20 & 4.60 \\
\hline 4400 & -3.9 & -0.07 & -0.04 & -0.04 & - & - & - & - \\
4400 & -3.0 & 0.00 & 0.00 & 0.00 & - & - & - & - \\
4400 & -2.4 & 0.00 & 0.00 & 0.00 & - & - & - & - \\
4400 & -1.2 & 0.00 & 0.00 & 0.00 & - & - & - & - \\
4400 & -0.6 & 0.00 & 0.00 & 0.00 & - & - & - & - \\
4400 & 0.0 & 0.00 & 0.00 & 0.00 & - & - & - & - \\
& & & & & & & & \\
4800 & -3.9 & -0.11 & -0.08 & -0.04 & -0.01 & -0.01 & - & - \\
4800 & -3.0 & -0.02 & -0.02 & -0.02 & -0.01 & -0.01 & -0.01 & 0.00 \\
4800 & -2.4 & 0.00 & 0.00 & 0.00 & 0.00 & -0.01 & -0.01 & 0.00 \\
4800 & -1.2 & 0.00 & 0.00 & 0.00 & 0.00 & 0.00 & 0.00 & 0.00 \\
4800 & -0.6 & 0.00 & 0.00 & 0.00 & 0.00 & 0.00 & 0.00 & 0.00 \\
4800 & 0.0 & 0.00 & 0.00 & 0.00 & 0.00 & 0.00 & 0.00 & 0.00 \\
& & & & & & & & \\
5200 & -3.9 & -0.07 & -0.03 & 0.01 & 0.05 & 0.08 & 0.12 & 0.13 \\
5200 & -3.0 & -0.05 & -0.06 & -0.06 & -0.05 & -0.04 & -0.03 & -0.01 \\
5200 & -2.4 & -0.01 & -0.01 & -0.01 & -0.01 & -0.01 & -0.01 & -0.01 \\
5200 & -1.2 & 0.00 & 0.00 & 0.00 & 0.00 & -0.01 & -0.01 & -0.01 \\
5200 & -0.6 & 0.00 & 0.00 & 0.00 & 0.00 & 0.00 & 0.00 & 0.00 \\
5200 & 0.0 & 0.00 & 0.00 & 0.00 & 0.00 & 0.00 & 0.00 & 0.00 \\
& & & & & & & & \\
5600 & -3.9 & 0.05 & 0.08 & 0.11 & 0.13 & 0.14 & 0.14 & 0.15 \\
5600 & -3.0 & -0.07 & -0.08 & -0.09 & -0.08 & -0.06 & -0.04 & -0.01 \\
5600 & -2.4 & -0.01 & -0.03 & -0.03 & -0.04 & -0.04 & -0.04 & -0.03 \\
5600 & -1.2 & 0.00 & 0.00 & -0.01 & -0.01 & -0.01 & -0.01 & -0.01 \\
5600 & -0.6 & 0.00 & 0.00 & 0.00 & -0.01 & -0.01 & -0.01 & -0.01 \\
5600 & 0.0 & 0.00 & 0.00 & 0.00 & 0.00 & 0.00 & 0.00 & 0.00 \\
& & & & & & & & \\
6000 & -3.9 & 0.19 & 0.19 & 0.19 & 0.18 & 0.17 & 0.16 & 0.15 \\
6000 & -3.0 & -0.04 & -0.06 & -0.06 & -0.05 & -0.04 & -0.02 & 0.01 \\
6000 & -2.4 & -0.01 & -0.03 & -0.05 & -0.06 & -0.07 & -0.07 & -0.06 \\
6000 & -1.2 & 0.01 & 0.00 & -0.01 & -0.01 & -0.01 & -0.01 & -0.01 \\
6000 & -0.6 & 0.00 & 0.00 & 0.00 & -0.01 & -0.01 & -0.01 & -0.01 \\
6000 & 0.0 & 0.00 & 0.00 & 0.00 & 0.00 & 0.00 & 0.00 & 0.00 \\
\hline & & & & & & & &
\end{tabular}


Table 5. NLTE abundance corrections for the $10036 \mathrm{Sr}$ II line.

\begin{tabular}{|c|c|c|c|c|c|c|c|c|}
\hline $\begin{array}{l}T_{\text {eff }} \\
\log g \\
\end{array}$ & {$[\mathrm{Fe} / \mathrm{H}]$} & 2.20 & 2.60 & 3.00 & $\begin{array}{r}\Delta_{\text {NLTE }} \\
3.40 \\
\end{array}$ & 3.80 & 4.20 & 4.60 \\
\hline 4400 & -3.9 & -0.14 & -0.15 & -0.18 & - & - & - & - \\
\hline 4400 & -3.0 & -0.13 & -0.12 & -0.12 & - & - & - & - \\
\hline 4400 & -2.4 & -0.13 & -0.12 & -0.12 & - & - & - & - \\
\hline 4400 & -1.2 & -0.17 & -0.15 & -0.13 & - & - & - & - \\
\hline 4400 & -0.6 & -0.15 & -0.14 & -0.13 & - & - & - & - \\
\hline 4400 & 0.0 & -0.10 & -0.11 & -0.10 & - & - & - & - \\
\hline 4800 & -3.9 & -0.18 & -0.18 & -0.19 & -0.20 & -0.12 & - & \\
\hline 4800 & -3.0 & -0.13 & -0.13 & -0.12 & -0.12 & -0.12 & -0.09 & -0.08 \\
\hline 4800 & -2.4 & -0.15 & -0.14 & -0.13 & -0.12 & -0.10 & -0.09 & -0.05 \\
\hline 4800 & -1.2 & -0.19 & -0.17 & -0.15 & -0.12 & -0.09 & -0.07 & -0.06 \\
\hline 4800 & -0.6 & -0.17 & -0.16 & -0.14 & -0.12 & -0.09 & -0.07 & -0.05 \\
\hline 4800 & 0.0 & -0.11 & -0.12 & -0.11 & -0.10 & -0.08 & -0.07 & -0.05 \\
\hline 5200 & -3.9 & -0.19 & -0.19 & -0.19 & -0.19 & -0.17 & -0.15 & -0.13 \\
\hline 5200 & -3.0 & -0.13 & -0.12 & -0.12 & -0.12 & -0.11 & -0.10 & -0.09 \\
\hline 5200 & -2.4 & -0.14 & -0.14 & -0.13 & -0.12 & -0.10 & -0.09 & -0.08 \\
\hline 5200 & -1.2 & -0.20 & -0.19 & -0.16 & -0.13 & -0.10 & -0.08 & -0.06 \\
\hline 5200 & -0.6 & -0.19 & -0.18 & -0.16 & -0.13 & -0.10 & -0.07 & -0.05 \\
\hline 5200 & 0.0 & -0.13 & -0.13 & -0.12 & -0.11 & -0.09 & -0.07 & -0.05 \\
\hline 5600 & -3.9 & -0.16 & -0.15 & -0.14 & -0.12 & -0.10 & -0.06 & -0.03 \\
\hline 5600 & -3.0 & -0.10 & -0.10 & -0.10 & -0.10 & -0.09 & -0.07 & -0.05 \\
\hline 5600 & -2.4 & -0.12 & -0.11 & -0.10 & -0.09 & -0.08 & -0.07 & -0.06 \\
\hline 5600 & -1.2 & -0.20 & -0.19 & -0.17 & -0.14 & -0.11 & -0.08 & -0.06 \\
\hline 5600 & -0.6 & -0.19 & -0.19 & -0.17 & -0.15 & -0.12 & -0.08 & -0.06 \\
\hline 5600 & 0.0 & -0.14 & -0.14 & -0.14 & -0.13 & -0.11 & -0.08 & -0.06 \\
\hline 6000 & -3.9 & -0.08 & -0.06 & -0.04 & -0.02 & 0.01 & 0.04 & 0.07 \\
\hline 6000 & -3.0 & -0.06 & -0.06 & -0.05 & -0.05 & -0.03 & -0.02 & 0.01 \\
\hline 6000 & -2.4 & -0.08 & -0.07 & -0.06 & -0.06 & -0.05 & -0.04 & -0.03 \\
\hline 6000 & -1.2 & -0.17 & -0.16 & -0.14 & -0.12 & -0.10 & -0.08 & -0.06 \\
\hline 6000 & -0.6 & -0.18 & -0.18 & -0.17 & -0.15 & -0.12 & -0.09 & -0.06 \\
\hline 6000 & 0.0 & -0.14 & -0.15 & -0.14 & -0.14 & -0.12 & -0.09 & -0.07 \\
\hline
\end{tabular}

